EPJ Web of Conferences 59, 06001 (2013)

DOI: $10.1051 /$ epjconf/20135906001

(C) Owned by the authors, published by EDP Sciences, 2013

\title{
Advances in HYDRA and its applications to simulations of inertial confinement fusion targets
}

\author{
M.M. Marinak, G.D. Kerbel, J.M. Koning, M.V. Patel, S.M. Sepke, \\ M.S. McKinley, M.J. O'Brien, R.J. Procassini and D. Munro
}

Lawrence Livermore National Laboratory, Livermore, CA, USA 94550

\begin{abstract}
A new set of capabilities has been implemented in the HYDRA 2D/3D multiphysics inertial confinement fusion simulation code. These include a Monte Carlo particle transport library. It models transport of neutrons, gamma rays and light ions, as well as products they generate from nuclear and coulomb collisions. It allows accurate simulations of nuclear diagnostic signatures from capsule implosions. We apply it to here in a 3D simulation of a National Ignition Facility (NIF) ignition capsule which models the full capsule solid angle. This simulation contains a severely rough ablator perturbation and provides diagnostics signatures of capsule failure due to excessive instability growth.
\end{abstract}

\section{INTRODUCTION}

Simulations of indirect drive ignition target designs for the National Ignition Facility (NIF) must model asymmetries originating from a wide variety of sources. These include intrinsic drive asymmetries due to the illumination geometry, and extrinsic asymmetries resulting from pointing and power balance errors between the beams. Roughness on the shell surfaces seed hydrodynamic instabilities which can also degrade capsule performance. These include discrete features such as ice grooves, dust grains, unusually large isolated bumps and the fill tube used to inject fuel into the capsule. The growth factors due to hydrodynamic instabilities are sufficiently large that perturbations progress into the nonlinear saturated regime. Experiments and simulations have established that larger saturation amplitudes are obtained by symmetric 3D perturbation shapes [1-10]. In addition the various asymmetries can combine in a capsule implosion so that accurate treatment of the phases between them is essential. Treating accurately the effects of these asymmetries on capsule performance and their manifestation in simulated diagnostics requires 3D simulation [11]. Previously we reported results from 3-D integrated simulations of the full ignition target and high resolution simulations of a capsule over a limited solid angle [12-14]. Here we report the first high resolution 3D simulation of a full implosion of a NIF ignition capsule carried out over the full $4 \pi$ solid angle. The simulation includes a Monte Carlo treatment of the burn product transport, enabling simulation of neutron diagnostics.

\section{INDIRECTLY DRIVEN NIF IGNITION CAPSULE}

The recent addition into HYDRA of a Monte Carlo transport package for neutrons, gamma rays and charged particles allows simulations to resolve in both energy and angle the distribution of burn particles produced by the thermonuclear burn. The Monte Carlo transport module, called Arrakis, models burn products, including those produced by the three body triton-triton fusion reaction, and includes in flight reactions. The effects of temperature and fluid motion are treated. The output is used to model the full set of NIF particle diagnostics, such as the Neutron Time of Flight, Magnetic Recoil Spectrometer, Gamma

This is an Open Access article distributed under the terms of the Creative Commons Attribution License 2.0, which permits unrestricted use, distribution, and reproduction in any medium, provided the original work is properly cited. 


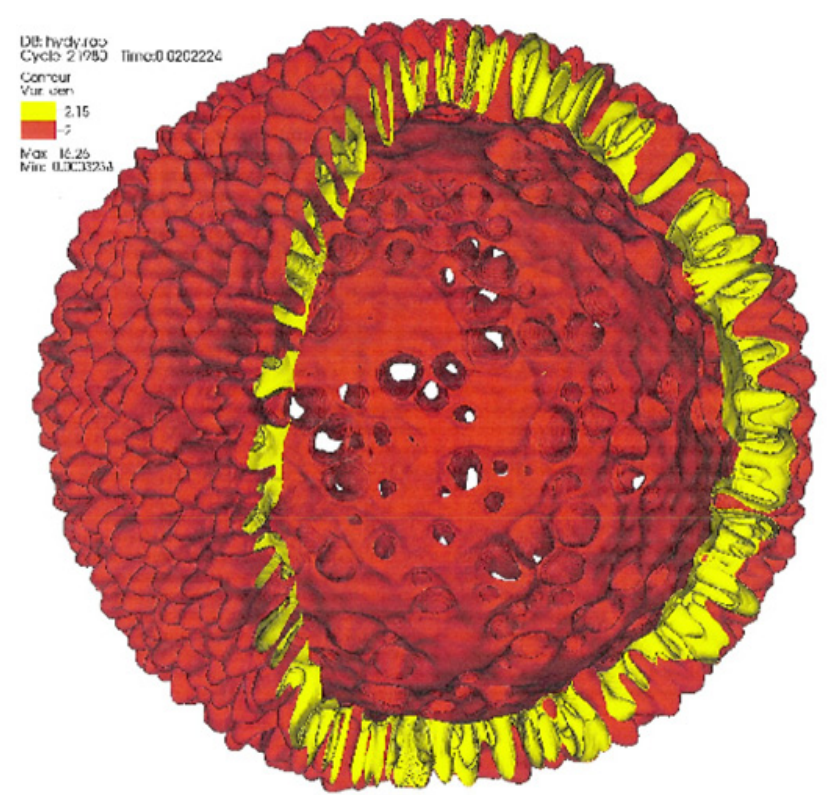

Figure 1. Cut away view of $2.0 \mathrm{~g} / \mathrm{cm}^{3}$ density contours in capsule shell at $20.22 \mathrm{nsec}$. Hohlraum axis is vertical.

Ray Histories and neutron imaging. Three dimensional simulations of the full capsule which include Monte Carlo transport of burn products enable us to generate the most accurate diagnostic signatures associated with various modes of capsule behaviour, including failure modes.

We consider a recent indirectly driven cryogenic NIF capsule design that employs a plastic ablator with germanium dopant arranged in layers of graded concentrations. The dimensions and compositions are as specified in Ref. [15]. The capsule-only simulation is performed using the radiation spectrum extracted from a 3D HYDRA integrated hohlraum simulation. This corresponds to the nominal drive as described in Ref. [15]. A drive asymmetry is imposed based upon spherical harmonic pressure moments extracted from the capsule in the integrated simulation. We initialize the capsule including roughness on the inner ice and outer ablator surfaces through intermediate modes $(\ell \leq 120)$. The roughness initialized on the inner ice surface is taken from the NIF specification scaled by 0.5 [16]. A severe roughness spectrum is initialized on the outer ablator. Compared to the NIF ablator spectrum specification its perturbation amplitudes are some 8 times larger. It has $60 \mathrm{~nm} \mathrm{rms}$ in the range of modes with $\ell>10$. In addition a $2 \mu \mathrm{m}$ peak to valley $\mathrm{P} 1$ thickness variation is imposed on the ablator, where P1 is the $1^{\text {st }}$ Legendre mode. With such severe surface roughness one would not expect the capsule to ignite. Rather this simulation will provide insight into the capsule behaviour with severe instability growth and provide the corresponding signatures for nuclear diagnostics.

The HYDRA capsule-only simulation was run on 4096 processors on a mesh of 57.9 million zones. At the peak 115 million Monte Carlo particles were transported. Figure 1 shows a cut away view of the density contours in the capsule shell at $20.22 \mathrm{nsec}$, when the capsule has converged to a radius of $\sim 350 \mu \mathrm{m}$.

The outer, ablator surface shows bubbles surrounded by narrow interconnecting spikes, characteristic of weakly nonlinear saturation of the Rayleigh-Taylor instability. The view through the capsule to the far side shows ablator bubbles are penetrating the shell, even before peak implosion velocity has been obtained. As the capsule converges further, and the shell decelerates, further instability growth causes the shell integrity to become severely compromised, with fuel streaming out of the bubbles. The hot spot is eventually quenched by many spikes of cold fuel, which are visible in Fig. 2. As a result the capsule 


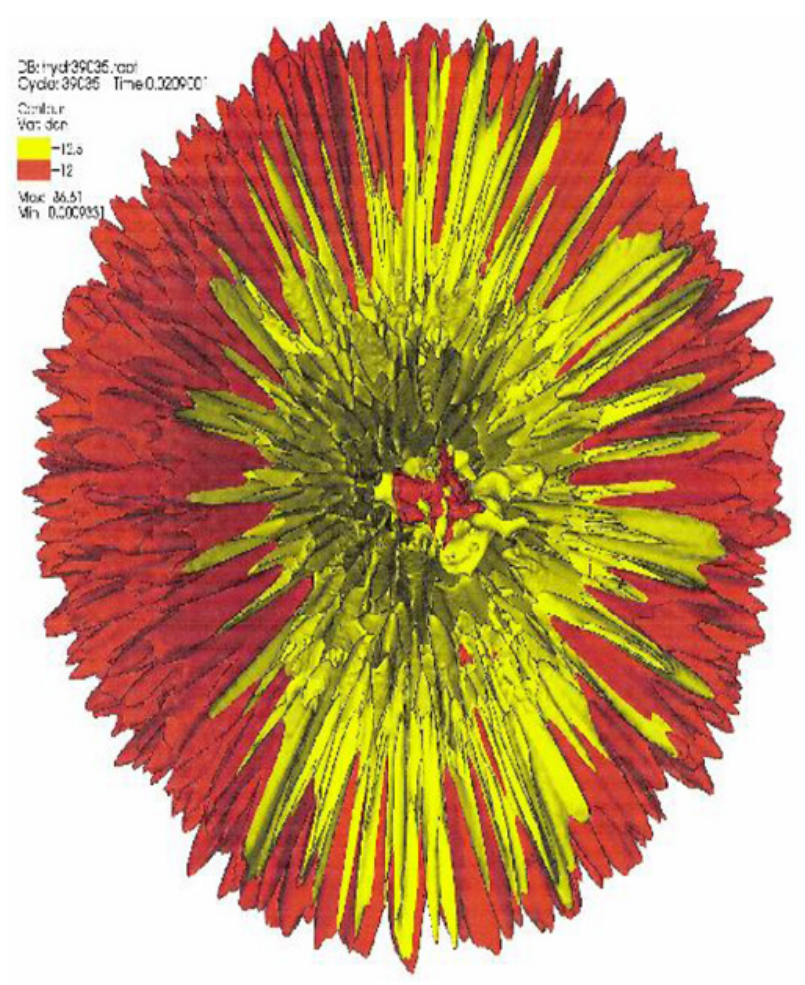

Figure 2. Cut away view of $12 \mathrm{~g} / \mathrm{cm}^{3}$ density contours in capsule shell at $20.9 \mathrm{nsec}$. Hohlraum axis is vertical.

does not ignite; the primary neutron yield obtained is $1.26 \times 10^{14}$. Detailed analysis of the neutron spectra produced show an average downscattered neutron fraction of 0.0272 , with a range of $0.02-$ 0.034. This compares with a downscattered fraction of 0.07 for a non-igniting clean 1-D simulation, indicating a substantial degradation in the capsule areal density $(\rho \mathrm{r})$. The neutron burn weighted ion temperature is depressed to $1.22 \mathrm{keV}$. Even though the capsule is subject to severe RT growth, the P1 asymmetry is clearly visible in the simulated neutron diagnostics. The neutron spectrum clearly shows the hot spot velocity is Doppler shifted by $30 \mathrm{~km} / \mathrm{sec}$, and in a direction which correlates with the orientation of the initial ablator thickness variation. The P1 is also visible in the downscattered neutron fraction. The neutron spectrum is distinguished by the large spectral shape distortion relative to a Gaussian. This indicates burn occurred over an unusually broad range of temperatures. The full width half maximum burn temperature directional variation, obtained from a 7-parameter fit, ranges from 1.08 to 1.40 , indicating significant non-spherical velocity. Since the neutron diagnostics are primarily sensitive to low mode asymmetries with $\ell \leq 3$, they give limited information on the spectral distribution of the non-spherical velocity.

While this simulation is not intended to model conditions of any given shot, the numerous particle diagnostics give us insight into the signatures of this failure mode. The techniques described here enable physicists to generate diagnostic signatures for the range of interesting failure modes using 3D HYDRA simulations. These also provide guidance for the diagnostic requirements to measure such effects. For example the P1 on this capsule would be detectable with $\sim 12$ detectors scattered around a solid angle measuring primary yield as long as the relative measurement accuracy is good to $+/-3 \%$. This is comparable to the accuracy of neutron activation detectors to be fielded soon on NIF. 
EPJ Web of Conferences

\section{SUMMARY}

This article described one advance in the modelling capabilities of the HYDRA code. This is one of several new types of capabilities that HYDRA has recently developed. With the implementation of a Monte Carlo neutron, gamma and charged particle transport package, diagnostic output can be generated for all NIF particle diagnostics from simulations. In conjunction with a new ability to perform highresolution capsule-only simulations treating the full capsule solid angle, this enables the most realistic, accurate assessment of the capsule implosion behaviour. The method outlined here enables physicists to study how various asymmetries grow and interact in 3D, and their ultimate impact upon ignition capsule performance. An example of a capsule with severely rough ablator gave distinctive diagnostic signatures due to excessive instability growth. This tool can be utilized to generate diagnostic signatures of various failure modes of interest.

This work was performed under the auspices of the U.S. Department of Energy by Lawrence Livermore National Security, LLC, Lawrence Livermore National Laboratory under Contract DE-AC52-07NA27344.

\section{References}

[1] J.W. Jacobs and I. Catton, J. Fluid Mech. 187, 353 (1988); 187, 329 (1988)

[2] G. Tryggvason and S.O. Unverdi, Phys. Fluids A 2, 656 (1990)

[3] T. Yabe, H. Hoshino, and T. Tsuchiya, Phys. Rev. A 44, 2756 (1991)

[4] H. Sakagami and K. Nishihara, Phys. Rev. Lett. 65, 432 (1990)

[5] R. P. J. Town and A. R. Bell, Phys. Rev. Lett. 67, 1863 (1991)

[6] J. Hecht, U. Alon, and D. Shvarts, Phys. Fluids 6, 12 (1994) ; D. Shvarts et al., Phys. Plasmas 2 , 2465 (1995)

[7] D. Layzer, Astrophys. J. 122, 1 (1955)

[8] J. P. Dahlburg and J. H. Gardner, Phys. Rev. A 41, 5695 (1990)

[9] J. P. Dahlburg et al., Phys. Fluids B 5, 571 (1993)

[10] M. M. Marinak et al., Phys. Rev. Lett. 75, 3677 (1995)

[11] M. M. Marinak et al., Phys. Rev. Lett. 80, 4426 (1998)

[12] M. M. Marinak et al., Phys. Plasmas, 5, 1125 (1998)

[13] M. M. Marinak et al., Phys. Plasmas, 8, 2275 (2001)

[14] S. W. Haan, et al., in proceedings of the Third International Conference on Inertial Fusion Science and Applications 2003, 55 (American Nuclear Society, B. A. Hammel et al., 2003)

[15] D. S. Clark, et al., Phys. Plasmas, 18, 082701 (2011)

[16] S. W. Haan, et al., Phys Plasmas, 18, 051001 (2011) 